\title{
Atividade larvicida de taninos isolados de Magonia pubescens St. Hil. (Sapindaceae) sobre Aedes aegypti (Diptera, Culicidae)
}

\author{
Larvicidal activity of tannins isolated of Magonia pubescens St. Hil. ( Sapindaceae) \\ against Aedes aegypti ( Diptera, Culicidae)
}

\section{Heloísa Helena Garcia da Silva ${ }^{1}$, Ionizete Garcia da Silva ${ }^{1}$, Regina Maria Geris dos Santos ${ }^{2}$, Edson Rodrigues Filho ${ }^{2}$ e Carmeci Natalina Elias ${ }^{1}$}

\begin{abstract}
RESUM0
Apresenta-se, pela primeira vez, o estudo fitoquímico das frações larvicidas, isoladas da Magonia pubescens, monitorado pelo estudo de eficácia sobre larvas de $3^{\circ}$ estádio de Aedes aegypti, na busca de alternativas para 0 controle desse mosquito e obtenção de estruturas químicas passíveis de aprimoramento da atividade pela via sintética de outros derivados. As frações bioativas foram monitoradas quimicamente através de cromatografia de camada delgada, utilizando como revelador uma solução ácida de vanilina, e analisadas por ressonância magnética nuclear de hidrogênio e espectrometria de massas. Os bioensaios com as frações foram realizados em quintuplicata, à temperatura de $28 \pm 1^{\circ} \mathrm{C}$, $80 \pm 5 \%$ de umidade relativa e fotofase de 12 horas. As concentrações letais encontradas da fração MP- 9 , que apresentou o maior potencial larvicida, $\mathrm{CL}_{50}$ e $\mathrm{CL}_{90}$, foram de 3,1 e 36,6ppm, respectivamente. Todos os experimentos foram acompanhados por uma série controle, contendo o mesmo número de larvas.
\end{abstract}

Palavras-chaves: Taninos. Larvicida. Magonia pubescens. Aedes aegypti.

\begin{abstract}
Phytochemical study of the larvicidal fractions wich were carried out for the first time, isolated of the Magonia pubescens, monitored by the study of efficacy against the $3^{\text {rd }}$ larval instar of Aedes aegypti, in the search of alternatives for the control of that mosquito and to obtain structures susceptible to chemical improving of the activity for the synthetic via of other deriveds. The fractions with biological activity were monitored chemically through chromatography of thin layer, using as revealing a solution acid of vanillin, analyzed by nuclear magnetic resonance of hydrogen and spectrometry of masses. The bioassays with the fractions were accomplished wit five replications, controlled at the temperature of $28 \pm 1^{\circ} \mathrm{C}, 80 \pm 5 \%$ of relative humidity and $12 \mathrm{~h}$ light. The found lethal concentrations of the fraction that it presented the largest potential larvicidal, $\mathrm{MP}-9, \mathrm{LC}_{50}$ and $\mathrm{LC}_{90}$, were of 3,1 and 36,6ppm, respectively. All the experiments were accompanied by a control series, containing the same number of larvae.
\end{abstract}

Key-words: Tannins. Larvicidal. Magonia pubescens. Aedes aegypti.

Aedes aegypti (Lin,1762) é atualmente 0 mosquito que apresenta a maior dispersão em áreas urbanas no mundo. A importância médica dessa espécie está na sua capacidade vetorial dos quatro sorotipos do vírus da dengue e do vírus amarílico. As altas densidades estão relacionadas ao comportamento sinantrópico e ao hábito antropofílico dessa espécie. Além disso, sua atividade hematofágica diurna e a complexidade dos centros urbanos têm maximizado os problemas do combate por aplicações espaciais de inseticidas ${ }^{27}$. Contudo, a perspectiva de controle desse vetor, na atualidade, esbarra na sua grande capacidade adaptativa a condições adversas, como 0 desenvolvimento em águas poluídas ${ }^{26}$, e a quiescência dos ovos em ambientes inóspitos ${ }^{24}$.

\footnotetext{
1. Laboratório de Biologia e Fisiologia de Insetos e Xenodiagnóstico do Instituto de Patologia Tropical e Saúde Pública da Universidade Federal de Goiás, Goiânia, G0. 2.Departamento de Química da Universidade Federal de São Carlos, São Carlos, SP.

Apoio Financeiro: CNPq, FAPESP, CAPES, OPAS, FUNAPE.

Endereço para correspondência: Dr. Ionizete Garcia da Silva. Dept ${ }^{0}$ Microbiologia, Imunologia, Parasitologia e Patologia/IPTSP/UFG, Caixa Postal 131, 74001-970 Goiânia, G0.

Tel: 5562 209-6128; Fax:55 62 261-2077.

e-mail: ionizete@ iptsp.ufg.br

Recebido para publicação em 31/5/2004

Aceito em 11/6/2004
} 
Uma das estratégias para combater a dengue é a eliminação do vetor através de produtos inseticidas. Como conseqüência do uso continuado desses produtos surgiram as populações resistentes ${ }^{313}$ de A. aegypti. Além disso, efeitos indesejáveis desses inseticidas como a permanência por longos períodos de tempo no meio ambiente, afetando os ecossistemas, estimularam a pesquisa de produtos naturais. Alguns estudos apontam compostos de origem botânica com atividade larvicida e potencial para uso no controle 571015161923282933 de vetores.

A Magonia pubescens St. Hil. (Sapindaceae) é uma planta característica do Cerrado brasileiro ${ }^{11}{ }^{12}$, apresenta grande densidade em áreas de terreno fraco e tem sido aproveitada na construção civil.

Estudos anteriores com extratos brutos etanólicos da M. pubescens mostraram atividade larvicida para A aegypti e A. albopictus. Mesmo sabendo que as plantas constituem uma fonte incomparável de compostos químicos inovadores, trabalhos envolvendo atividade biológica ou toxicológica de princípios ativos puros ${ }^{182333}$ são em números inexpressivos, sendo a maioria das publicações referentes à atividade de extratos brutos ${ }^{79} 10282932$ ou frações ${ }^{6} 1528$. Na bibliografia pertinente, não foram encontrados registros envolvendo os estudos sobre os componentes químicos da $\mathrm{M}$ pubescens. Como existe atualmente uma exigência de que produtos de origem natural sejam quimicamente estudados ${ }^{4}$, para evidenciar sua eficácia e segurança de seu uso, este trabalho apresenta pela primeira vez, 0 estudo fitoquímico da M pubescens, monitorado pelo estudo de eficácia sobre larvas de A aegypti, na busca de alternativas para 0 controle desse mosquito e obtenção de estruturas químicas passíveis de aprimoramento da atividade pela via sintética de outros derivados.

\section{MATERIAL E MÉTODOS}

Material vegetal. As cascas do caule de M pubescens foram coletadas na região de Alto Paraíso - Chapada dos Veadeiros - Goiás, no mês de novembro de 2001, e trazidas ao Laboratório de Bioatividade de Plantas, IPTSP/UFG.

Extração e fracionamento. As cascas foram secas em estufa de ventilação forçada, a $40^{\circ} \mathrm{C}$, e moídas até baixa granulometria. Em seguida, $1,4 \mathrm{~kg}$ do pó foi extraído por percolação com etanol ( 72 horas), por duas vezes consecutivas, à temperatura ambiente, e 0 extrato bruto foi filtrado e concentrado em evaporador rotativo $(60,2 g)$. Após os resultados de atividade larvicida do extrato bruto etanólico (e.b.e.) ${ }^{28}{ }^{30}$, sobre A aegypti, cerca de $51,2 \mathrm{~g}$ deste material foi submetido à cromatografia em coluna utilizando como fase móvel os solventes n-hexano, diclorometano e metanol, seguindo a técnica de gradiente de polaridade. Foram obtidas nove frações, as quais foram codificadas como MP-1 a MP-9.

Bioensaios. Para a detecção da atividade larvicida nas frações foram utilizadas larvas de $3^{\circ}$ estádio de A. aegypti, criadas de acordo com metodologia já definida ${ }^{25}$. Uma solução-mãe foi preparada com uma determinada quantidade da fração de interesse, pré-solubilizada em dimetilsulfóxido
(DMSO) e dissolvida em água, numa quantidade suficiente para obter a concentração de $1000 \mathrm{ppm}$. A partir desta solução, a série de diluições foi preparada para obter soluções de concentrações desejadas. Em $25 \mathrm{~mL}$ de cada uma dessas soluções foram adicionadas 20 larvas. Os bioensaios foram realizados em quintuplicata, a $28 \pm 1^{\circ} \mathrm{C}, 80 \pm 5 \%$ de umidade relativa e fotofase de $12 \mathrm{~h}$. Todos os experimentos foram acompanhados de uma série controle, contendo o mesmo número de larvas em DMSO e água destilada. Os dados obtidos da mortalidade $\mathrm{x}$ concentração (ppm) foram analisados pelo programa SAEG ( Sistema de Análises estatísticas), em gráfico de Probit, para determinar as concentrações letais $\left(\mathrm{CL}_{50 \text { e } 90}\right)$ e os respectivos intervalos de confiança.

Análise das frações bioativas. As frações bioativas foram monitoradas quimicamente através de cromatografia de camada delgada (CCD), utilizando como revelador uma solução ácida de vanilina. Além disso, essas amostras foram analisadas por ressonância magnética nuclear de hidrogênio ( RMN $^{1} \mathrm{H}$ ) em um espectrômetro Bruker ARX-200, utilizando como solvente clorofórmio deuterado $\left(\mathrm{CDCl}_{3}\right)$ ou metanol deuterado ( $\left.\mathrm{CD}_{3} \mathrm{OD}\right)$, e tetrametilsilano (TMS) como padrão interno de referência. A análise das frações por espectrometria de massas foi realizada em um espectrômetro QuattroLC Micromass, utilizando como fonte de ionização ESI/APCI nos modos negativo e positivo.

\section{RESULTADOS E DISCUSSÃO}

Das nove frações obtidas a partir do e.b.e. da $\mathrm{M}$ pubescens apenas as frações MP-7, MP-8 e MP-9 apresentaram atividade tóxica sobre larvas de $3^{\circ}$ estádio de A. aegypti (Tabela 1 ). Com a partição dos extratos e separação cromatográfica foi possível obter concentrações letais extremamente baixas em relação aos estudos anteriormente feitos com 0 e.b.e. ${ }^{28}$. Contudo, este apresentou a vantagem de ser hidrossolúvel, simplificando o preparo da solução para uso.

Tabela 1 - Atividade larvicida das frações ativas de Magonia pubescens sobre larvas de terceiro estádio de Aedes aegypti.

\begin{tabular}{lcr}
\hline Frações & $\mathrm{CL}_{50}(\mathrm{IC} 95 \%) \mathrm{ppm}$ & $\mathrm{CL}_{90}(\mathrm{IC} 95 \%) \mathrm{ppm}$ \\
\hline $\mathrm{MP}_{7}$ & $67,1(60,9-71,1)$ & $95,5(90,7-104,1)$ \\
$\mathrm{MP}_{8}$ & $39,7(22,5-26,4)$ & $380,7(250,6-477,3)$ \\
$\mathrm{MP}_{9}$ & $3,1(0,08-6,79)$ & $36,6(25,9-89,1)$ \\
\hline
\end{tabular}

IC $95 \%$ - Intervalo de confiança a 95\% de probabilidade.

Obs.: não houve morte no grupo controle

Estudos que já evidenciaram potencialidade de uso de frações botânicas para 0 controle de $\mathrm{A}$ aegypti, apontam $\mathrm{CL}_{50}$ variando de 0,1 a 49ppm ${ }^{16} 233133$, o que permite considerar que a MP-9 apresentou resultado coerente com a literatura.

As análises por CCD das frações MP-7 (diclorometano:metanol, 6:4), MP-8 (diclorometano:metanol, 4:6) e MP-9 (metanol) revelaram manchas avermelhadas após revelação com a vanilina, sugerindo a presença de derivados fenólicos, mais precisamente, taninos. A formação de coloração vermelha, resultante da 
complexação do agente revelador vanilina com os taninos, é um método fácil e rápido para diagnosticar a presença dessas substâncias em extratos e frações, embora ainda não conclusivo para a identificação da classe a qual os taninos pertencem ${ }^{22}$.

Adicionalmente, essas frações também foram submetidas a análises de prospecção para definir a classe de taninos ${ }^{14}$ presentes, utilizando-se a solução de cloreto férrico. Na presença dessa solução, os taninos hidrolisáveis produzem uma forte coloração azul, enquanto que os taninos condensados ou catéquicos exibem uma forte coloração verde ${ }^{34}$. Nossos testes químicos indicaram a presença de taninos catéquicos na fração MP-9. Os dados de RMN para essa fração corroboraram os testes químicos. Os espectros de RMN ${ }^{1} \mathrm{H}$ para MP-9 contêm vários multipletos com deslocamentos químicos para hidrogênios aromáticos entre 6,00 e 8,00ppm, os quais caracterizam os grupos catequinas nestes taninos. Neste mesmo espectro foram também observados sinais para uma série de hidrogênios carbinólicos ( $\delta 3,00$ a 4,50) correspondendo a hidrogênios $\mathrm{H}-2 \mathrm{e}$ H-3, bem como a hidrogênios de unidades de carboidratos indicando que parte das catequinas podem estar ocorrendo na forma de glicosídeos. Os espectros de massas obtidos por electrospray nos modos positivos e negativos, mostraram que a fração MP-9 é composta de uma mistura bastante complexa de substâncias. No entanto, uma das substâncias apresentou $[\mathrm{M}+\mathrm{H}]^{+}$a m/z 865 e [M-H] a m/z 863. Assim, a partir das análises por espectrometria de massas, uma das substâncias pode ser identificada como uma proantocianidina na forma trimérica massa molecular $=864 \mathrm{Da}$ ). A estrutura molecular para essa substância, que foi a fração mais promissora da M pubescens, é mostrada na Figura 1.

As proantocianidinas são largamente distribuídas na natureza, em plantas altas, particularmente coníferas, e são também encontradas em muitos produtos alimentícios ${ }^{8}$ como chás, cacau e sorgo.

Taninos são substâncias fenólicas, solúveis em água, com massa molecular entre 500 e 3000Da. Muitas vezes, são os princípios ativos de plantas empregadas na medicina tradicional para 0 tratamento de diversas moléstias orgânicas, por apresentarem atividades biológicas como a ação bactericida ${ }^{21}$, fungicida, moluscicida e inibição enzimática ${ }^{34}$. Os taninos condensados são oligômeros e polímeros formados pela policondensação de duas ou mais unidades flavanoídicas e possuem grande importância biológica por suas fortes interações com íons metálicos e macromoléculas como os polissacarídeos, além de apresentar a habilidade para formar complexos solúvei ${ }^{34} \mathrm{com}$ alcalóides, gelatinas e diversas proteínas. Essa habilidade que os taninos exibem para interagir com proteínas torna essa classe de substâncias bastante tóxica ${ }^{34}$ a insetos, fungos e bactérias.

Estudos realizado $\mathrm{s}^{20}$ através de cortes histológicos, mostraram 0 efeito tóxico do ácido tânico, um tanino natural hidrolisável, sobre o epitélio do intestino médio de larvas de dípteros. Outros autores ${ }^{17}$, após avaliarem os efeitos tóxicos de taninos sobre a fauna associada aos culicíneos, sugeriram que os taninos vegetais podem ser úteis como complemento em programas de controle de espécies de mosquitos

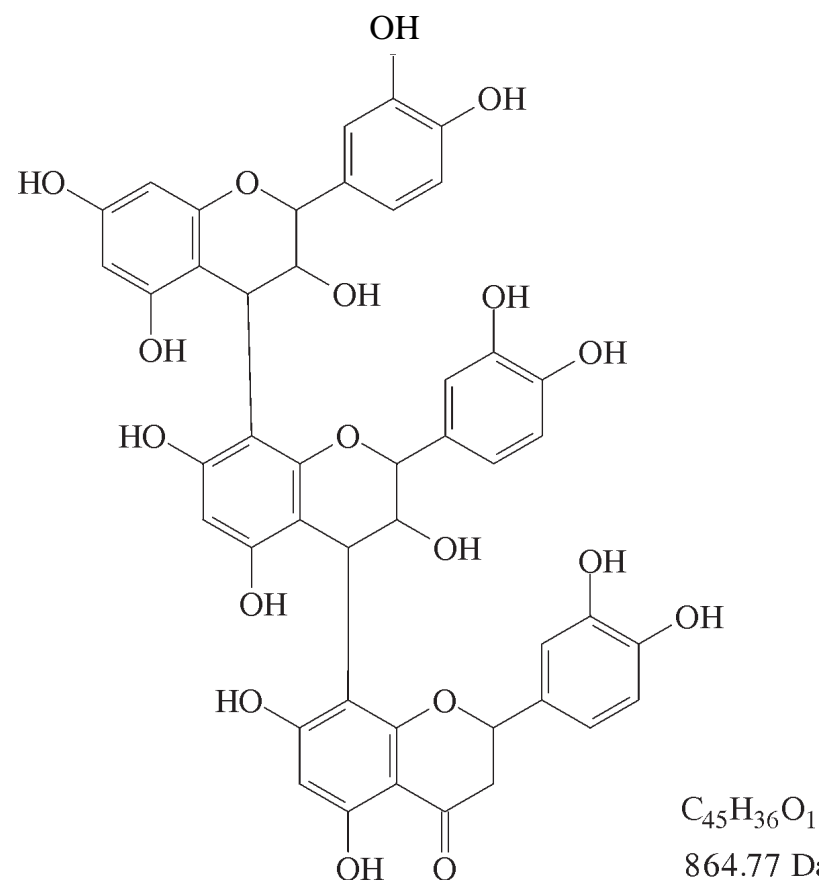

Figura 1 - Estrutura molecular do tanino catéquico identificado na fração larvicida MP-9, obtida de Magonia pubescens.

associados à atividade humana. Mais especificamente sobre a $\mathrm{M}$ pubescens e anteriores à partição, fracionamento e identificação do tanino catéquico, estudos ${ }^{12}{ }^{28}$ foram realizados com 0 extrato, evidenciando os mecanismos de ação e as alterações morfológicas que provocaram as mortes nas larvas de A aegypti, sendo similares àquelas registradas pelo ácido tânico ${ }^{28}$. Além disso, estudos toxicológicos ${ }^{28}$ do e.b.e. realizados em coelhos, ratos e cobaios mostraram-se atóxicos de acordo com as normas para produtos vegetais. Testes de campo evidenciaram sua degradação e atoxicidade a partir da quarta semana ${ }^{28}$. Mesmo com essas informaç̧ões favoráveis, estudos toxicológicos adicionais com 0 tanino catéquico serão feitos para avaliar seu impacto, principalmente em espécies não alvo, e de formulações que dêem estabilidade e praticidade no uso desse fitoinseticida potencial.

\section{REFERÊNCIAS BIBLIOGRÁFICAS}

1. Arruda W, Oliveira GMC, Silva IG. Alterações morfológicas observadas em larvas de Aedes aegypti (Linnaeus, 1762) submetidas à ação do extrato bruto etanólico da casca do caule da Magonia pubescens. Entomologia y Vectores 10:47-60, 2003a.

2. Arruda W, Oliveira GMC, Silva IG. Toxicidade do extrato etanólico de Magonia pubescens sobre larvas de Aedes aegypti. Revista da Sociedade Brasileira de Medicina Tropical 36:17-25, 2003b.

3. Carvalho LAF, Silva IG. Avaliação longitudinal da atividade do temephós a $1 \%$ sobre 0 Aedes aegypti (Lin,1762). Entomologia y Vectores 7:191-201, 2000.

4. Cascón V, Gilbert B. Caracterization of the chemical composition of oleoresins of Copaifera guianensis Desf., Copaifera duckei Dwyer and Copaifera multijuga Hayne. Phytochemistry 55: 773-778, 2000.

5. Choochote W, Kananapothi D, Panthong A, Taesotikul T, Jitpadi A, Chaithong U, Pitasawat B. Larvicidal, adulticidal and reppelents effects of Kaempferia galanga. Southeast Asian Journal of Tropical Medicine Public Health 30:470-476, 1999. 
6. Ciccia G, Coussio J, Mongelli E. Insecticidal activity against Aedes aegypti larvae of some medicinal South American plants. Journal Ethnopharmacology 72:185-189, 2000.

7. Consoli RAGB, Mendes NM, Pereira JP, Santos BS, Lamounier MLA. Influência de diversos derivados de vegetais na sobrevida das larvas de Aedes fluviatillis em laboratório. Memórias do Instituto Oswaldo Cruz 83:87-93,1988.

8. De Bruyne T, Pieters L, Deelstra H, Vietinck A. Condensed vegetable tannins: Biodiversity in structure and biological activities. Biochemical Systematics and Ecology 27:445-449, 1999.

9. Dharmashktu NS, Prabhakaran PK, Menon PK. Laboratory study on the mosquito larvicidal properties of leaf and seed extract of the plant Agave americana. Journal of Tropical Medicine and Hygiene 90:7982,1987.

10. Guimarães VP, Silva IG, Silva HHG, Rocha C. Atividade larvicida do extrato bruto etanólico de Magonia pubescens St. Hil. sobre Aedes albopictus (Skuse,1894) (Diptera, Culicidae). Revista de Patologia Tropical 34:159165, 2001.

11. Lorenzi H. Árvores Brasileiras: Manual de identificação e cultivo de plantas arbóreas nativas do Brasil. Plantarum, Nova Odessa, SP, 1992.

12. Laboriau MLS. A semente de Magonia pubescens St. Hil. - Morfologia e germinação. Anais da Academia Brasileira de Ciências 45: 501-536, 1973.

13. Macoris MLG, Camargo MF, Silva IG, Takaku L, Andrighetti MT. Modificação da suscetibilidade de Aedes aegypti ao Temephós. Revista de Patologia Tropical 24:31-40, 1995.

14. Matos FjA. Introdução à Fitoquímica Experimental. Edições da Universidade Federal do Ceará, Fortaleza, CE, p.43, 1997.

15. Monzon RB, Alvior JP, Luczon LL, Morales AS, Mutuc FE. Larvicidal potential of five Philippine plants against Aedes aegypti (Linnaeus) and Culex quinquefasciatus ( Say). Southeast Asian Journal Tropical Medicine Public Health 25:755-759,1994.

16. Park IK, Lee SG, Shin SC, Park JD, Ahn YJ. Larvicidal activity of isobutylamides identified in Piper nigrum fruits against three mosquito species. Journal of Agricultural and Food Chemistry 50:1866-1870, 2002.

17. Pautou MP, Rey D, David JP, Meyran JC. Toxicity of vegetable tannins on crustacea associated with Alpine mosquito breeding sites. Ecotoxicology and Environmental Safety 47:323-332, 2000 .

18. Pinto AC, Silva DHS, Bolzani VS, Lopes NP, Epifanio RA. Produtos naturais: atualidades, desafios e perspectivas. Química Nova 25:45-61, 2002.

19. Pizarro AP, Oliveira-Filho AM, Parente JP, Melo MT, Santos CE, Lima PR. Aproveitamento do resíduo da indústria do sisal no controle de larvas de mosquitos. Revista da Sociedade Brasileira de Medicina Tropical 32:23-29, 1999

20. Rey D, Pautou MP, Meyran JC. Histopathological effects of tannic acid on the midgut epithelium of some aquatic diptera larvae. Journal of Invertebrate Pathology 73:173-181, 1999.
21. Scalbert A. Antimicrobial properties of tannins. Phytochemistry 30: 3875 3883, 1991

22. Schofield P, Mbugua DM, Pell NA. Analysis of condensed tannins: a review. Animal Feed Science and Technology 91:21-40, 2001.

23. Siddiqui BS, Asfhan F, Ghiasuddin FS, Navqi SN, Tariq RM. Two inseticidal tetranortriterpenoids from Azadirachta indica. Phytochemistry 53:371. 376, 2000.

24. Silva HHG, Silva IG. Influência do período de quiescência dos ovos sobre o ciclo evolutivo de Aedes aegypti (Linnaeus, 1762) (Diptera, Culicidae) em condições de laboratório. Revista da Sociedade Brasileira de Medicina Tropical 32:349-355, 1999.

25. Silva HHG, Silva IG, Lira KS. Metodologia de criação, manutenção de adultos e estocagem de ovos de Aedes aegypti (Linnaeus, 1762) em laboratório. Revista de Patologia Tropical 27:51-63, 1998.

26. Silva HHG, Silva IG, Oliveira CLNS, Elias CN. Adaptação do Aedes aegypti (Linnaeus,1762) em criadouros artificiais com áqua poluída. Entomologia y Vectores 6:383-391, 1999.

27. Silva HHG, Silva IG, Souza SS, Guimarães VP, Elias CN, Pimenta JF. Estudo comparativo de eficiência das técnicas de ultra baixo volume (UBV) e termonebulização ( FOG) no controle de Aedes aegypti. Informe Epidemiológico do Sistema Único de Saúde 10:45-46, 2001.

28. Silva IG, Guimarães VP, Lima CG, Silva HHG, Elias CN, Mady CM, Silva VVM, Nery AP, Rocha KR, Rocha C, Isac E. Efeito larvicida e toxicológico do extrato bruto etanólico da casca do caule de Magonia pubescens sobre Aedes aegypti (Diptera, Culicidae) em criadouros artificiais. Revista de Patologia Tropical 32:73-86, 2003.

29. Silva IG, Santos AH, Ferri PH, Alves RBN, Melo RQ, Peixoto L, Silva HHG, Elias CN, Isac E, Lira KS, Camargo MF. Ação larvicida do extrato bruto etanólico de Magonia pubescens St.Hil. ( tingui-do-cerrado) , sobre o Aedes aegypti ( Lin.) em laboratório. Revista de Patologia Tropical 25:51-59, 1996.

30. Silva IG, Silva HHG, Guimarães VP, Lima GC, Pereira AL, Filho ER, Rocha C. Prospecção da atividade inseticida de plantas do Cerrado, visando ao combate de Aedes aegypti. Informe Epidemiológico do SUS 10:51-52, 2001.

31. Simas NK, Lima EC, Conceição SR, Kuster RM, Oliveira Fo AM. Produtos naturais para 0 controle da transmissão de dengue - atividade de Myroxylon balsamum (óleo vermelho) e de terpenóides e fenilpropanóides. Química Nova 27:46-49, 2004

32. Sivagnaname N, Kalyanasundaram M. Laboratory evaluation of methanolic extract of Atlantia monophyla (Family: Rutaceae) against immature stages of mosquitoes and non-target organisms. Memórias do Instituto Oswaldo Cruz 99:115-118, 2004.

33. Slimestad R, Marston A, Mavi A, Hostettmann K. Larvicidal constituents of Melantheria albinervia. Planta Medica 61:562-563, 1995.

34. Simões CMO, Schenkel EP, Gosmann G, Mello JCP, Mentz LA, Petrovick PR. Farmacognosia: da planta ao medicamento. Editora da Universidade Federal do Rio Grande do Sul, Porto Alegre, 2001. 